\title{
TAROT: Observing gamma-ray bursts "in progress"
}

\author{
M. Boër ${ }^{1}$, M. Bringer ${ }^{1}$, A. Klotz ${ }^{1}$, A.M. Moly ${ }^{1}$, D. Toublanc ${ }^{1}$, G. Calvet $^{2}$, J. Eysseric ${ }^{2}$, A. Leroy ${ }^{2}$, M. Meissonnier $^{2}$, \\ R. Malina ${ }^{3}$, P. Sanchez ${ }^{3}$, C. Pollas ${ }^{4}$, and H. Pedersen ${ }^{5}$ \\ 1 Centre d'Études Spatiales des Rayonnements (CESR/CNRS), BP. 4346, 31028 Toulouse Cedex 04, France \\ 2 Institut National des Sciences de l'Univers (CNRS), Division Technique, 77 Av. Denfert Rochereau, 75014 Paris, France \\ 3 Laboratoire d'Astronomie Spatiale (LAS/CNRS), BP. 8, 13376 Marseille Cedex 12, France \\ 4 Observatoire de la Côte d'Azur, 2130 route de l'Observatoire, Caussols, 06460 St Vallier de Thiey, France \\ 5 Copenhagen University Observatory, Juliane Maries Vej 30, 2100 Copenhagen E, Denmark
}

Received January 13; accepted March 12, 1999

\begin{abstract}
The primary objective of the Télescope à Action Rapide pour les Objets Transitoires (TAROT Rapid Action Telescope for Transient Objects) observatory is the detection of cosmic Gamma-Ray Burst sources at optical wavelengths while still active in Gamma-rays. It features a very rapid slewing mount, a $25 \mathrm{~cm}$ aperture telescope with a $2 \times 2 \mathrm{deg}$. fov, and is able to reach the 17 th $V$ magnitude in 10 s. A powerful scheduling algorithm, and an automated data processing system makes TAROT a fully autonomous facility. TAROT entered into service during the fall of 1998.
\end{abstract}

Key words: gamma-ray bursts — telescopes

\section{Introduction}

Until now half a dozen of Gamma-Ray Bursts (hereafter GRBs) have been detected at optical wavelengths, usually several hours at least after the detection of the GammaRay Burst event itself, because of the time needed for the Beppo-SAX team to get and analyse the data from the satellite. On the other hand, CGRO/BATSE produces a large number of source positions, though they are difficult to use because of their large error radii. The intermediate case will be HETE-II, which will produce 25 moderately accurate localizations per year. At present we can state that the from the time starting at the GRB onset, and for few hours, GRB optical counterparts are almost a terra incognita.

The goal of the Télescope à Action Rapide pour les Objets Transitoires (TAROT-1, Rapid Action Telescope for Transient Objects) is to fill this observationnal gap, and to acquire data while the GRB source is still active at high energy wavelengths. TAROT-1 is a fully automated observatory, able to preset rapidly to any point of the sky upon a request sent by the GRB Coordinate Network (GCN; Barthelmy et al. 1999), and then to perform the observation of the GRB source error box.

Send offprint requests to: M. Boër

Correspondence to: Michel.Boer@cesr.fr
Since we get an alert about every 15 days, there is ample time to address secondary science objectives. Most of it will be used to perform a complete survey of the sky accessible from TAROT-1, used as a reference for the detection of new or variable objects in subsequent frames. Other objectives range from the detection of supernovae, eventually associated with GRBs, to the detection and followup of variable objects. In this paper we present the main characteristics of the TAROT-1 experiment, the first data acquired from it, and the perspectives of development.

\section{The instrument}

TAROT-1 features a large field of view telescope, in association with a fast slewing, fully automated mount. The main characteristics of the instrumentation are summarized in Table 1.

The currently used camera was manufactured by APOGEE Corp., and includes a KAF 1300 Kodak $1024 \times 1280$ CCD chip. This results in a field of view of $1 \times 1.2 \mathrm{deg}$. We are currently developping a new camera based on a Thomson CCD, whose characteristics are outlined in Table 1, which will be available in May 1999. Its main advantages are a larger fov (4 sq. deg.), a quicker readout time $(1 \mathrm{~s})$, and low readout and thermal noises. TAROT-1 entered into operation during the fall of 1998, at the Calern station of the Observatoire de la Côte d'Azur (France).

\section{Software and operations}

Five software modules have been implemented in order to achieve a high degree of automation for TAROT-1 (Bringer et al. 1999). The REQUESTOR and MAJORDOME softwares are in charge of the request processing and of the scheduling of the telescope, using an optimised timeline (Bringer et al. 1999). The CONTROL software is in charge of the control and monitoring of the telescope, and sends orders to the CAMERA software, which in turn produces a FITS frame with the raw data. The TAITAR software produces a list of sources, astrometrically and photometrically calibrated. 
Table 1. Summary of the TAROT-1 main instrumental characteristics

\begin{tabular}{|c|c|}
\hline Aperture & $25 \mathrm{~cm}, \mathrm{f} / 3.3$ \\
\hline Field of view & $2 \mathrm{deg} \times 2 \mathrm{deg}$ \\
\hline Optical Resolution & $20 \mu \mathrm{m}$ \\
\hline Mount type & Equatorial \\
\hline $\begin{array}{l}\text { Maximum slewing time } \\
\text { to target }\end{array}$ & $3 \mathrm{~s}$ \\
\hline $\begin{array}{l}\text { Maximum speed } \\
\text { (slew) }\end{array}$ & $120 \mathrm{deg} / \mathrm{s}$ \\
\hline Tracking speed & Adjustable $0-60 \mathrm{deg} / \mathrm{s}$ \\
\hline $\begin{array}{l}\text { Max. acceptable wind } \\
\text { speed }\end{array}$ & $80 \mathrm{~km} / \mathrm{h}$ \\
\hline CCD type & Thomson full frame \\
\hline CCD size & $3 \times 3 \mathrm{~cm}, 2048 \times 2048$ pixels \\
\hline Pixel size & $15 \mu \mathrm{m}$ \\
\hline $\mathrm{CCD}$ readout noise & \\
\hline $\begin{array}{l}@-50^{\circ} \mathrm{C} \\
\mathrm{CCD} \text { readout time }\end{array}$ & $\leq 10 \mathrm{e}^{-}$ \\
\hline $\begin{array}{l}\text { (slow mode) } \\
\text { CCD Readout time }\end{array}$ & $1 \mathrm{~s}$ \\
\hline (fast modes) & 0.5 and $0.25 \mathrm{~s}$ \\
\hline $\begin{array}{l}\text { Typical integration time } \\
\text { (alert) }\end{array}$ & $10-20 \mathrm{~s}$ \\
\hline $\begin{array}{l}\text { Typical integration time } \\
\text { (routine) }\end{array}$ & $60 \mathrm{~s}$ \\
\hline Maximum integration time & $300 \mathrm{~s}$ \\
\hline Filter wheel & open, $V, R, I, B+V, R+I$ \\
\hline $\begin{array}{l}\text { Limiting } V \text { magnitude } \\
\text { in } 10 \mathrm{~s}\end{array}$ & 17 \\
\hline
\end{tabular}

TAROT functions in either of two operating modes. In routine mode, requests from the database are normally processed, following a timeline computed by the MAJORDOME. Whenever an alert occurs (e.g. a GRB position from the GCN), the alert mode is activated, and the telescope slews in less than $3 \mathrm{~s}$ (depending on the amplitude of the move), to the new target. Including the time needed by the GCN to compute the source position (4.5 s; Barthelmy 1998), and the INTERNET delay $(0.5 \mathrm{~s})$, TAROT begins its observations $8 \mathrm{~s}$ after the burst onset. At the same time, follow-up observations are inserted in the timeline for the remaining of this alert night and the following nights and a new schedule is computed by the MAJORDOME. BATSE error boxes will be observed as a mosaic of observations, a process which will take less than five minutes with the next camera. However, about once a year, it will happen that the right source position will be observed during the first observation, resulting in a simultaneous BATSE/TAROT observation. For the other satellites, including HETE-II, the fov of TAROT-1 is large enough to contain the full error box. This software chain allows TAROT-1 to produces without any human intervention, an image of the area of interest, including a list of sources, their variability, extension, etc. in few minutes. These products

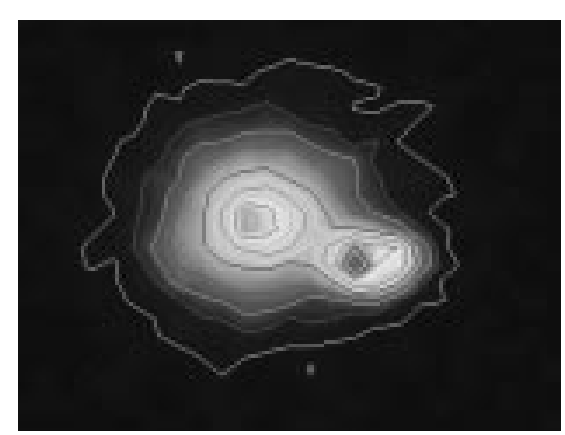

Fig. 1. SN 1998aq and its host galaxy, NGC 3982. The image was taken in June 1998, when the supernovae was at $V$ magnitude 14

will be rapidly disseminated, in order to allow for fast follow-up observations at larger facilities.

Another interesting feature of TAROT-1 is its ability to compute a map of the cloud coverage of the sky, from the observation of evenly spaced reference stars. This greatly enhance the throughput of TAROT-1, since TAROT can observe even if we have a partial coverage, or a region free of clouds. In any case, the large fov of TAROT allows the validation of each individual image, even when the sky is partially covered.

\section{Conclusion and perspectives}

Figure 1 displays an image of SN 1998aq seen over the background of its host galaxy NGC 3982 when it was at $m_{V}=14$. This image is typical of the capabilities of TAROT to detect an object even in high background conditions. The next major step of TAROT-1 will be the implementation of new routines for the recognition of objects, on the data processing side, and of the new camera on the instrument side. As soon as this detector will be in service, we will perform a survey of the accessible sky, in order to build a reference database, which will be used to search for new or variable objects. At present we are validating our data processing routines, in order to provide an automated alert service to selected parties. We study also a new, enhanced version of the MAJORDOME.

Acknowledgements. The TAROT-1 project is funded by the Centre National de la Recherche Scientifique, Institut National des Sciences de l'Univers, on the French side, and by the Carlsberg Fundation on the Danish side. The authors are also very grateful to Dr. S. Barthelmy, who initiated and maintains the GRB Coordinate Network, a system used extensively by TAROT-1. Thanks are also due to Prof. B. Paczyńsky for stimulating discussions on the observation of GRBs at optical wavelengths.

\section{References}

Barthelmy S., 1998, the GCN (BACODINE) system, on line document available at http://gcn.gsfc.nasa.gov/gcn/ gcn_describe.html

Barthelmy S., et al., 1999, A\&AS (in press)

Bringer M., Peignot C., Boër M., Fontan G., Merce C., 1999, A\&AS (in press) 